(C) Klemenov A.V., 2021

DOI 10.18019/1028-4427-2021-27-1-128-131

\title{
Possibilities with backward walking for knee pathology (literature review)
}

\author{
A.V. Klemenov
}

City Clinical Hospital № 30, Nizhny Novgorod, Russian Federation

\begin{abstract}
Introduction Recently backward/retro walking has been increasingly used in medicine. Kinetic and kinematic analysis during backward walking showed advantages over traditional ambulation making it ideal for those who struggle with knee pain. Retro walking is well known for reducing the strain on the knees and it is one of the few natural ways to strengthen the quadriceps of the thigh. Walking backwards leads to a more significant load on the cardiovascular and respiratory systems. This load builds a more substantial increase in both the aerobic and anaerobic capabilities than just walking forward with similar parameters of physical activity. Material and methods A systematic search of studies published during the period up to February 2020 was conducted using bibliographic databases in English, including Medline, PubMed, Scopus, Web of Science and in Russian, including eLIBRARY, CyberLeninka to summarize the available information on the possibilities of retro walking in treatment and rehabilitation of patients with knee pathology. Search terms included 'backward/retro walking/running', 'knee joint'. Results and discussion Several studies have been performed to explore the effectiveness of backward walking for knee osteoarthritis. There is a strong evidence that backward walking can be a useful adjunct to conventional physiotherapy to improve pain, knee function and strength of the quadriceps muscles. There is a limited number of papers describing effects of backward walking for patellofemoral pain syndrome and anterior cruciate ligament injury. Conclusions Although there is little data overall reporting effects of backward walking, and more studies are needed to further explore this topic this version of walking has been shown to provide great beneficial effects in knee joint rehabilitation. Keywords: backward walking, rehabilitation, knee joint, anterior cruciate ligament, osteoarthritis, patellofemoral pain syndrome, literature review
\end{abstract}

\section{INTRODUCTION}

The knee joint is the largest and most complex joint in the human body with the greatest range of movement at the gait and is subjected to severe functional stress and pressure. Functions of the knee joint include flexion and extension, providing mobility for the leg and maintaining balance and support the body in an upright position, act as a shock absorber, facilitating maximum stability and withstanding large forces. Knee functioning requires a complex anatomical organization to ensure adequate articulations by the femur, tibia and patella. The complexity of the structure, variety of functions and the loads experienced predispose the knee to a greater risk of injuries and diseases being common for musculoskeletal pathologies. About $15 \%$ of trauma patients admitted to hospitals are those suffering from knee problems, and knee pain remains the most common reason for visits to surgeons, traumatologists and therapists. Knee pain and stiffness are most common reasons people seek out an orthopedic and trauma surgeon or general practitioner. Different approaches including surgical and nonsurgical, medical and non-medical methods are used to treat various pathologies of the knee joint with physical therapy integrated in the treatment of any pathology of the joints. There is strong evidence for the efficacy of physical therapy among drug free options.
An overview of systematic reviews and meta-analyses show the effectiveness of different physical activity and exercise interventions in reducing pain severity and its impact on function in a variety of joint pathology [1-6].

Walking is the most physiological type of physical exercise allowing to improve the function of the heart and lungs, increase muscle mass and strengthen bones, achieve psychological well-being toning up the body. Recently backward/retro walking has been increasingly used in medicine. Biomechanical analysis during backward walking showed advantages over traditional ambulation. Walking backwards leads to a more significant load on the cardiovascular and respiratory systems [7, 8] and builds a more substantial increase in both the aerobic and anaerobic capabilities than just walking forward with similar parameters of physical activity $[9,10]$. Retro walking is well known for reducing the strain on the knees [9, 11 ] and it is one of the few natural ways to strengthen the quadriceps of the thigh [12-15]. The role of retrowalking has been shown to be important in the knee rehabilitation programs.

The purpose of the work was to investigate the benefits of retro walking in treatment and rehabilitation of patients with knee disorders. 


\section{MATERIAL AND METHODS}

A systematic search of studies published during the period up to February 2020 was conducted using bibliographic databases in English, including Medline, PubMed, Scopus, Web of Science and in Russian, including eLIBRARY, CyberLeninka to summarize the available information on the possibilities of retro walking in treatment and rehabilitation of patients with knee pathology. Search terms included 'backward/ retro walking/running', 'knee joint'. Full-text copies of potentially relevant studies were analyzed.

\section{RESULTS}

The search strategy generated 13 citations as relevant to randomized controlled clinical trials studying the effectiveness of retrowalking in a variety of knee pathologies including osteoarthritis of the knee joint $(n=10)$, injuries to the anterior cruciate ligament $(n=2)$, patellofemoral pain syndrome $(n=1)$. Other publications included in the review were meant to explore biomechanical aspects of backward walking providing pathogenetic rationale for the use of the method in the knee rehabilitation programs.

\section{DISCUSSION}

Osteoarthritis (OA) is the most common joint disease in the world affecting more than $10 \%$ of the world's population [16-19]. Knee OA/gonarthrosis is the most common OA localization and is characterized by chronic pain, impaired function, early disability and reduced quality of life. Results of randomized clinical trials demostrate high rehabilitation potential of retrowalking in patients with knee OA [20-29]. Retrowalking is evaluated as an adjunct to conventional treatment (exercise therapy and/or physiotherapy) versus conventional treatment alone. A. Alghadir et al. compared the effect of retrowalking on pain, functional disability, quadriceps muscle strength and physical performance in 68 individuals with knee OA randomized into three groups of 6-week retro versus forward walking program versus control group [20]. The retro walking group had a greater reduction in pain intensity and functional disability measured by the WOMAC, and a greater improvement in the quadriceps muscle strength than the control group. Statistically significant improvement of knee pain and functional disability due to retrowalking have been reported in other series [21-26, 28, 29]. Because backward walking eliminates the typical heel-strike to the ground with the main propulsion and shock absorption ankle putting far less impact on the knees, it can lead to pain improvement $[17,23,28,30]$. Walking backwards is well known for reducing the strain on the knees and requires less range of motion from the knee joints [21, $23,28,29]$. Effects of retro walking on pain, balance and functional performance have been reported in knee $\mathrm{OA}$ [21, 23-29]. Improved functional performance due to walking backwards training is associated with pain relief and altered joint biomechanics, limited adduction moment, in particular [21, 28, 29].

Greater increases in the power of the quadriceps femoris, a powerful stabilizer muscle of the knee joint at exercise/rest was reported in subjects engaged in backward walking [17, 30, 31]. Quantitative changes of the quadriceps strength are also associated with improved functional outcomes and pain in patients with knee OA [32]. Statistically significant improvement in the power of the quadriceps femoris was related to the effects of backward gait training protocol on knee adduction moment and impulse in patients with knee OA [21-23]. Forward walking and backward walking along with conventional therapy were found to be equally effective and not better than conventional treatment alone in reducing pain and improving physical function and quality of life $[20,27]$. There have been no publications available to compare backward walking and different types of physical exercises in patients with knee OA. The metaanalysis of seven randomized clinical trials performed by T. Balasukumaran et al. in 2019 [33] demonstrated significant values in favour of backward walking with conventional physiotherapy treatment reducing pain and functional disability and improving quadriceps strength in patients suffering from knee OA.

As OA is most common in adults and the elderly, pain arising from the patellofemoral joint and known as patellofemoral pain syndrome (PFPS) or runner's/jumper's knee is seen frequently in young and physically active people. The majority of patients with frank patellofemoral instability would experience lateral movement of the patella traveling through the trochlear groove as the knee nears full extension. Painful overstretching of the structures supporting the patella results in impaired biomechanics of the patellofemoral joint, increased compressive forces and subsequent articular cartilage wear. The quadriceps femoris muscle imbalance are deemed to be involved with the patella deviating laterally at terminal extension as it exits the trochlear 
groove [34-36]. The beneficial effects of backward gait can be used in treatment and rehabilitation of patients with PFPS with muscle activation patterns in lower limbs being different at comparison of forward and backward walking. O. Abdelraouf et al. examined the effect of backward walking and forward walking on the myoelectric activity ratio of the quadriceps femoris muscle in 20 females with PFPS and 20 healthy controls [37]. During backward walking, the vastus medialis oblique activity was significantly higher in PFPS patients with the vastus medialis oblique/vastus lateralis ratio (1/1) being close to ideal in PFPS patients.

Sprains and tears of the anterior cruciate ligament (ACL), one of the main stabilizing ligaments of the knee, resisting anterior tibial translation and internal tibial rotation are among the most common knee injuries. ACL injuries have been getting steadily more common in the world of professional sports and fitness activities that can put stress on the knee. The majority of ACL reconstructions performed for sports injury are associated with inevitable immobilization with resultant loss of quadriceps power, cardiorespiratory fitness level which is unlikely to permit a return to serious sports. A systematic rehabilitation program that would emphasize the return to symmetrical knee motion, including hyperextension, is necessary to achieve the optimum result of ACL reconstructions [38, 39]. Patients who underwent stretching or ACL reconstruction and practiced a systematic rehabilitation exercise program with backward walking demonstrate improved quadriceps strength recovery preventing ACL overstretching. Backward walking is also associated with increased cardiopulmonary load compared to forward walking, and therefore can be used to increase aerobic capacity in athletes who require reduced knee load due to knee injury. M. Shen et al. and F. Zampeli et al. reported backward walking as a rehabilitation technique improving knee proprioception and knee function after ACL reconstruction [40, 41]. S. Rezazadeh et al. implemented a controller on a powered knee-ankle prosthesis and tested with a transfemoral amputee subject who could successfully perform a wide range of periodic and non-periodic tasks including backward walking [42].

\section{CONCLUSION}

The knee joint is an important part in the kinetic chain of the lower extremity. The complexity of the structure and a variety of functions predispose the knee to a greater risk of injury. Physical therapy exercises including walking as the most accessible form of physical activity are essential for treatment and rehabilitation of patients with knee pathologies. Retrowalking has recently emerged as a new concept in physiotherapy and rehabilitation of knee conditions in terms of improving quadriceps strength as a significant contributing factor to recovery from patellofemoral symptoms while simultaneously increasing aerobic capacity and decreasing joint stress. Although more studies are needed to further explore this topic retrowalking has been shown to provide great beneficial effects in knee joint rehabilitation.

\section{REFERENCES}

1. Kelley G.A., Kelley K.S., Callahan L.F. Aerobic Exercise and Fatigue in Rheumatoid Arthritis Participants: A Meta-Analysis Using the Minimal Important Difference Approach. Arthritis Care Res. (Hoboken), 2018, vol. 70, no. 12, pp. 1735-1739. DOI: 10.1002/acr.23570

2. Geneen L.J., Moore R.A., Clarke C., Martin D., Colvin L.A., Smith B.H. Physical activity and exercise for chronic pain in adults: an overview of Cochrane Reviews. Cochrane Database Syst. Rev., 2017, vol. 4, no. 4, pp. CD011279. DOI: 10.1002/14651858.CD011279.pub3

3. Goh S.L., Persson M.S.M., Stocks J., Hou Y., Welton N.J., Lin J., Hall M.C., Doherty M., Zhang W. Relative efficacy of different exercises for pain, function, performance and quality of life in knee and hip osteoarthritis: systematic review and network meta-analysis. Sports Med., 2019, vol. 49, no. 5, pp. 743-761. DOI: 10.1007/s40279-19-01082-0

4. Tanaka R., Ozawa J., Kito N., Moriyama H. Efficacy of strengthening or aerobic exercise on pain relief in people with knee osteoarthritis: a systematic review and meta-analysis of randomized controlled trials. Clin. Rehabil., 2013, vol. 27, no. 12, pp. 1059-1071. DOI: 10.1177/0269215513488898

5. Alzahrani H., Mackey M., Stamatakis E., Pinheiro M.B., Wicks M., Shirley D. The effectiveness of incidental physical activity interventions compared to other interventions in the management of people with low back pain: A systematic review and meta-analysis of randomised controlled trials. Phys. Ther. Sport., 2019, vol. 36, pp. 34-42. DOI: 10.1016/j.ptsp.2018.12.008

6. Sharif K., Watad A., Bragazzi N.L., Lichtbroun M., Amital H., Shoenfeld Y. Physical activity and autoimmune diseases: Get moving and manage the disease. Autoimmun. Rev., 2018, vol. 17, no. 1, pp. 53-72. DOI: 10.1016/j.autrev.2017.11.010

7. Terblanche E., Page C., Kroff J., Venter R.E. The effect of backward locomotion training on the body composition and cardiorespiratory fitness of young women. Int. J. Sports Med., 2005, vol. 26, no. 3, pp. 214-219. DOI: 10.1055/s-2004-820997

8. Thomas K.S., Hammond M., Magal M. Graded forward and backward walking at a matched intensity on cardiorespiratory responses and postural control. Gait Posture, 2018, vol. 65, pp. 20-25. DOI: 10.1016/j.gaitpost.2018.06.168

9. Hoogkamer W., Meyns P., Duysens J. Steps forward in understanding backward gait: from basic circuits to rehabilitation. Exerc. Sport Sci. Rev., 2014, vol. 42, no. 1, pp. 23-29. DOI: 10.1249/JES.0000000000000000

10. Kachanathu S.J., Alenazi A.M., Algarni A.D., Hafez A.R., Hameed U.A., Nuhmani S., Melam G. Effect of forward and backward locomotion training on anaerobic performance and anthropometrical composition. J. Phys. Ther. Sci., 2014, vol. 26, no. 12, pp.1879-1882. DOI: 10.1589/jpts.26.1879

11. Roos P.E., Barton N., van Deursen R.W. Patellofemoral joint compression forces in backward and forward running. J. Biomech., 2012, vol. 45, no. 9, pp. 1656-1660. DOI: 10.1016/j.jbiomech.2012.03.020

12. Hao W.Y., Chen Y. Backward walking training improves balance in school-aged boys. Sports Med. Arthrosc. Rehabil. Ther. Technol., 2011, vol. 3 , pp. 24. DOI: $10.1186 / 1758-2555-3-24$ 
13. Cha H.G., Kim T.H., Kim M.K. Therapeutic efficacy of walking backward and forward on a slope in normal adults. J. Phys. Ther. Sci., 2016, vol. 28, no. 6, pp. 1901-1903. DOI: 10.1589/jpts.28.1901

14. Lamb T., Yang J.F. Could different directions of infant stepping be controlled by the same locomotor central pattern generator? J. Neurophysiol., 2000 , vol. 83 , no. 5 , pp. $2814-2824$. DOI: $10.1152 /$ jn.2000.83.5.2814

15. Schneider C., Lavoie B.A., Capaday C. On the origin of the soleus H-reflex modulation pattern during human walking and its task-dependent differences. J. Neurophysiol., 2000, vol. 83, no. 5, pp. 2881-2890. DOI: 10.1152/jn.2000.83.5.2881

16. Kloppenburg M., Berenbaum F. Osteoarthritis year in review 2019: epidemiology and therapy. Osteoarthritis Cartilage, 2020, vol. 28, no. 3 , pp. 242-248. DOI: $10.1016 /$ j.joca.2020.01.00

17. Vina E.R., Kwoh C.K. Epidemiology of osteoarthritis: literature update. Curr. Opin. Rheumatol., 2018, vol. 30, no. 2, pp. 160-167. DOI: 10.1097/ BOR.0000000000000479

18. Johnson V.L., Hunter D.J. The epidemiology of osteoarthritis. Best Pract. Res. Clin. Rheumatol., 2014, vol. 28, no. 1, pp. 5-15. DOI: 10.1016/j. berh.2014.01.004

19. Neogi T., Zhang Y. Epidemiology of osteoarthritis. Rheum. Dis. Clin. North. Am., 2013, vol. 39, no. 1, pp. 1-19. DOI: 10.1016/j.rdc.2012.10.004.

20. Alghadir A.H., Anwer S., Sarkar B., Paul A.K., Anwar D. Effect of 6-week retro or forward walking program on pain, functional disability, quadriceps muscle strength, and performance in individuals with knee osteoarthritis: a randomized controlled trial (retro-walking trial). BMC Musculoskelet. Disord., 2019, vol. 20, no. 1, pp. 159. DOI: 10.1186/s12891-019-2537-9

21. Gondhalekar G.A., Deo M.V. Retrowalking as an adjunct to conventional treatment versus conventional treatment alone on pain and disability in patients with acute exacerbation of chronic knee osteoarthritis: a randomized clinical trial. N. Am. J. Med. Sci., 2013, vol. 5, no. 2, pp. 108-112. DOI: $10.4103 / 1947-2714.107527$

22. Khyatee, Mittal K., Gupta S.K. Retro treadmill walking as a rehabilitative tool in knee pain and quadriceps insufficiency. International Journal of Research in Science and Technology, 2013, vol. 2, no. 4, pp. 1-8.

23. Rathi M., Palekar T., Varghese A. Efficacy of backward walking on patients with osteoarthritis of knee on quadriceps strength, pain and physical functions. Indian J. Physiother. Occup. Ther., 2014, vol. 8, no. 4, pp. 192-196.

24. Anadkat H., Ajith S., Dhanesh Kumar K.U. Effectiveness of retro walking treadmill training on pain and disability in knee osteoarthritis: a randomized controlled trail. Int. J. Pharm. Bio. Sci., 2015, vol. 6, no. 4, pp. 43-50.

25. Manisha N., Joginder Y., Priyanka R. Effect of retro walking on pain, balance and functional performance in osteoarthritis of knee. Indian J. Physiother. Occup. Ther., 2015, vol. 9, no. 3, pp. 154-159.

26. Somashekar R.R., Sridharamurthy J.N., Timsina S., Jha V. A study to compare the effectiveness of transcutaneous electrical nerve stimulation with retro-walking versus ultrasound therapy with retro-walking in chronic osteoarthritis of knee. J. Evolution Med. Dent. Sci., 2015, vol. 4, no. 60, pp. 10494-10503. DOI: 10.14260/jemds/2015/1512

27. Rangey P.S., Sheth M.S., Vyas N.J. Comparison of effectiveness of forward and backward walking on pain, physical function, and quality of life in subjects with osteoarthritis of knee. Int. J. Health Allied Sci., 2016, vol. 5, no. 4, pp. 220. DOI: 10.4103/2278-344X.194085

28. Wadhwa D.N., Hande D.N. Effects of retro walking on osteoarthritis of knee in geriatric population. IOSR J. Sports Phys. Educ. (IOSR-JSPE), 2016, vol. 3, no. 2, pp. 37-43. DOI: 10.9790/6737-03023743 www.iosrjournals.org 37

29. Shankar P., Bhandiwad R.M.A., Pai H. Effectiveness of retrowalking in chronic osteoarthritis of knee joint. Innovative Journal of Medical and Health Science, 2013, vol. 3, no. 1.

30. Lee M., Kim J., Son J., Kim Y. Kinematic and kinetic analysis during forward and backward walking. Gait Posture, 2013, vol. 38, no. 4, pp. $674-678$. DOI: $10.1016 /$ j.gaitpost.2013.02.014

31. Riskowski J.L., Mikesky A.E., Bahamonde R.E., Alvey T.V. 3rd, Burr D.B. Proprioception, gait kinematics, and rate of loading during walking: are they related? J. Musculoskelet. Neuronal Interact., 2005, vol. 5, no. 4, pp. 379-387.

32. Hasan S. Quadriceps Femoris Strength Training: Effect of Neuromuscular Electrical Stimulation vs Isometric Exercise in OA of Knee. Indian J. Physiother. Occup. Ther., 2015, vol. 9, no. 3, pp. 129-134. DOI: 10.5958/0973-5674.2015.00110.0

33. Balasukumaran T., Olivier B., Ntsiea M.V. The effectiveness of backward walking as treatment for people with gait impairments: a systematic review and meta-analysis. Clin. Rehabil., 2019, vol. 33, no. 2, pp. 171-182. DOI: 10.1177/0269215518801430

34. Fagan V., Delahunt E. Patellofemoral pain syndrome: a review on the associated neuromuscular deficits and current treatment options. Br. J. Sports Med., 2008, vol. 42, no. 10, pp. 789-795. DOI: $10.1136 / \mathrm{bjsm} .2008 .046623$

35. Crossley K.M., van Middelkoop M., Barton C.J., Culvenor A.G. Rethinking patellofemoral pain: Prevention, management and long-term consequences. Best Pract. Res. Clin. Rheumatol., 2019, vol. 33, no. 1, pp. 48-65. DOI: 10.1016/j.berh.2019.02.004

36. Gaitonde D.Y., Ericksen A., Robbins R.C. Patellofemoral Pain Syndrome. Am. Fam. Physician, 2019, vol. 99, no. 2, pp. 88-94.

37. Abdelraouf O.R., Abdel-Aziem A.A., Ahmed A.A., Nassif N.S., Matar A.G. Backward walking alters vastus medialis oblique/vastus lateralis muscle activity ratio in females with patellofemoral pain syndrome. Turk. J. Phys. Med. Rehabil., 2019, vol. 65, no. 2, pp. 169-176. DOI: 10.5606/ tftrd.2019.2445

38. Shelbourne K.D., Klotz C. What I have learned about the ACL: Utilizing a progressive rehabilitation scheme to achieve total knee symmetry after anterior cruciate ligament reconstruction. J. Orthop. Sci., 2006, vol. 11, no. 3, pp. 318-325. DOI: 10.1007/s00776-006-1007-z

39. Aglietti P., Giron F., Buzzi R., Biddau F., Sasso F. Anterior cruciate ligament reconstruction: Bone-patellar tendon-bone compared with double semitendinosus and gracilis tendon grafts. A prospective, randomized clinical trial. J. Bone Joint Surg. Am., 2004, vol. 86, no. 10, pp. $2143-2155$.

40. Shen M., Che S., Ye D., Li Y., Lin F., Zhang Y. Effects of backward walking on knee proprioception after ACL reconstruction. Physiother. Theory Pract., 2019, pp. 1-8. DOI: 10.1080/09593985.2019.1681040

41. Zampeli F., Moraiti C.O., Xergia S., Tsiaras V.A., Stergiou N., Georgoulis A.D. Stride-to-stride variability is altered during backward walking in anterior cruciate ligament deficient patients. Clin. Biomech. (Bristol, Avon), 2010, vol. 25, no. 10, pp. 1037-1041. DOI: 10.1016/j.clinbiomech.2010.07.015

42. Rezazadeh S., Quintero D., Divekar N., Gregg R.D. A Phase Variable Approach to Volitional Control of Powered Knee-Ankle Prostheses. Rep. U. S., 2018, pp. 2292-2298. DOI: 10.1109/IROS.2018.8594023

Received: 23.03 .2020

\section{Information about the author:}

Aleksey V. Klemenov, M.D., Ph.D.,

City Clinical Hospital № 30, Nizhny Novgorod, Russian Federation,

Email:klemenov_av@list.ru 